\title{
Evolution of fatigue crack corrosion from surface irregularities
}

\author{
A. P. Jivkov* \\ Malmö University, Division of Solid Mechanics, SE-205 06 Malmö, Sweden
}

\begin{abstract}
A moving boundary model is presented for crack nucleation and growth from surface flaws. It concerns with chemical attack that results in material dissolution. A controlling mechanism for evolution is the rupture of a brittle corrosion-protective film that is built up along the corroding surface. The evolution rate is a function of the degree of protective film damage caused by the surface straining. The problem is formulated for an elastic body containing a single and double pits. Low-frequency cyclic loading is considered. Numerical solution is proposed. The behaviours of a growing crack and of two competing cracks are described. Stages of incubation, blunting and steady-state growth characterise a single crack evolution. The steady-state growth rate is found independent of the initial geometry. Stages of independent growth, interactive growth and arrest of one crack characterise the evolution of two competing cracks. The lengths of the arrested cracks are presented as functions of the ratio between the pit depth for a series of different distances between the pits. It is emphasized that the solutions correspond to a homogeneous material. Further work is required to account for the material microstructure.
\end{abstract}

Keywords: Crack evolution; Moving boundary; Corrosion pitting; Fatigue corrosion cracking; Finite elements.

\section{Introduction}

Fatigue is one of the physical processes that leads to materials degradation and failure of engineering components and structures. It has been a subject of experimental and analytical research for more than a century [1]. Aggressive environment introduces corrosion and adds to further degradation of the material and shortens the life of structural members [2-7]. A widely used hypothesis for the environment assisted crack growth is that of dissolution [8,9] which is localized at the surface region where the metal is exposed and experiences the highest strains. Electrochemistry models [10-12] assume stress corrosion crack growth, with attention focused on the crack tip region. A single parameter such as the crack tip opening displacement, or the stress intensity factor can be used. A model for strain-driven corrosion cracking has been proposed in [13]. The model assumes a moving boundary problem. A finite part of the crack tip region was considered as susceptible to corrosion. A similar approach is used in this work to investigate the growth of surface pits involving crack formation under cyclic

\footnotetext{
*Fax: +46-40-6657135; E-mail: andrey.jivkov@ts.mah.se
} 
loading. The existence of corrosion pits has been found to nucleate fatigue corrosion cracks [14-18]. Experiments with pre-existing corrosion pits have shown that the fatigue cracks always initiate from the pits [16]. Moreover, the reduction of the specimen lifetime has been found to depend on the pre-existing pit depth. It has been shown [18] that the effect of corrosion by pitting on fatigue life can be related to the stress concentration factor at the root of the pit [14]. An equivalent stress intensity factor for a corroded pit was used to formulate a criterion for the transition from pitting to fatigue cracking. Proposed in [15] is an empirical relation for estimating the reduced fatigue life as a function of the initial pit depth. All these works show that crack formation starts at the early stages of fatigue. The work in [17] considers the entire corrosion fatigue process as that consisting of three steps: pit development, corrosion fatigue short crack growth (usually in mode II), and corrosion fatigue long crack growth (usually in mode I). Suggested is that strain-driven dissolution is responsible for the pit development and short crack growth. A synergetic approach is therefore needed where the mechanical straining of the surface causes material dissolution and strain redistribution.

\section{Evolution model}

Consider a non-linear elastic solid body with surface exposed to corrosive environment. Prior to load application, a passive film is assumed to cover densely the contact surface and prevent any dissolution. The bulk material of the body and the material of the passive film are considered as two phases of the same material with different constitutive behaviours. The passive film is assumed to be brittle in tension and to deform elastically together with the bulk material up to eventual film rupture. The film thickness does not enter into the model. One additional material parameter is sufficient to describe the behaviour of the film. The strain at film rupture, denoted by $\varepsilon_{f}$, is accepted as a suitable mechanical characteristic. Figure 1 shows schematically a portion of the surface containing a flaw before and after load application. The load is applied parallel to the contact surface and at large distance from the flaw. The magnitude is considered to be small enough so as not to break the film along the flat parts of the surface but sufficient to create stress concentration at the flaw where the film breaks. When the load increases, the passive film deforms elastically until the strain parallel to the surface reaches the rupture strain, $\varepsilon_{f}$. After that the film breaks at full load, a number of film fragments separated by gaps of bare metal are created. The unprotected gaps are exposed to the corrosive solution and the metal dissolves. This advances the portion of the surface with destroyed protection. The advancement occurs normally to the surface at a rate, denoted by $v_{s}$. The discrete process may be "smeared" to assume a continuous formulation. Assume that the film fragments relax after the rupture. If $S$ is the length of a surface arc before the deformation, and $s$ denotes its deformed length, the fraction of the gaps created along the arc is accepted as definition for surface strain, or film damage measure: $\varepsilon=(s-S) / s$, Figure 1 . The continuous formulation assumes that the strain must be larger than the rupture strain of the film in order to have metal exposed to corrosion. The advancement rate of a surface point, $r$, may be written as

$$
r=v \varepsilon H\left(\varepsilon-\varepsilon_{f}\right),
$$


where $v$ is a corrosion rate obtained for a unstrained bare surface, and $H(x)$ is the Heaviside step function, i.e. $H(x)=1$ if $x>0$, and $H(x)=0$ otherwise. In the absence of mechanical straining, growth rates adopt a power low dependence on time in the majority of electrochemical experiments. An expression for the corrosion rate $v$ as a function of time $t$ has been proposed [19]:

$$
v=\left\{\begin{array}{ll}
v_{0} & \text { for } t \leq t_{0} \\
v_{0}\left(\frac{t_{0}}{t}\right)^{m} & \text { for } t>t_{0}
\end{array},\right.
$$

where $v_{0}, t_{0}$ and $m$ are electrochemical parameters, which depend on the solid-solution composition and reflect the dissolution and repassivation kinetics and the transport processes in the environment. The exponent in eq. (2) is physically bounded in the interval $0<m<1$. Hence $v_{0}$ provides the maximum possible dissolution rate that may be observed under pure bare surface conditions.

The surface evolution model in eq. (1) considers strain-driven corrosion, which together with the mechanical equilibrium of the solid constitute a moving boundary problem. It offers a numerical solution to the moving boundary problem. For simplicity, the load is taken to be cyclic with rectangular shape. The period between the cycles is denoted by $T$ and the peak cycle duration by $T_{0}$. Then $\varepsilon$ represents the peak strain during a cycle. The average penetration advance per cycle at a surface point, denoted by $d$, could be found by inserting eq. (2) into (1) and integrating over the cycle. Introduce the function $\lceil x\rceil$, with the property $\lceil x\rceil=x$ when $x<1$ and $\lceil x\rceil=1$ otherwise. The result of the integration then reads

$$
d=d_{0} \varepsilon H\left(\varepsilon-\varepsilon_{f}\right)
$$

where

$$
d_{0}=v_{0} T_{0}\left(\frac{1}{1-m}\left\lceil\frac{t_{0}}{T_{0}}\right\rceil^{m}-\frac{m}{1-m}\left\lceil\frac{t_{0}}{T_{0}}\right\rceil\right) .
$$

Note that $d_{0}$ is a known constant, providing all the parameters entering eq. (4) are known and assumed constant. It offers the maximum possible surface advance per cycle, achieved under pure bare metal conditions. It is worth noting that eq. (3) represents the material loss of the solid during one cycle per unit surface area. Now the moving boundary problem, defined in terms of rate of surface evolution in eq. (1), may be replaced by a sequence of static problems solved for discretely changing boundary value problems, eq. (3).

\section{Problems statement}

Consider a plane body occupying the region $0 \leq X_{1} \leq B$ and $-\mathrm{B} \leq X_{2} \leq \mathrm{B}$ with respect to a fixed coordinate system $\left(X_{1}, X_{2}\right)$. The body surface at $X_{1}=0,-\mathrm{B} \leq X_{2} \leq \mathrm{B}$ is assumed in contact with a corrosive environment and initially covered by a passive film. The rupture strain of the passive film is $\varepsilon_{f}=0.001$. The maximum penetration advance is chosen as $d_{0}=0.001$. An actual value, obtained via eq. (4) for physically reasonable parameters should be used to scale the time, or number of cycles. On the contact surface, a single pit or two competing pits can be taken. They are initially covered by a passive film. Figure 2 shows the schematic geometries for two general cases: single pit and 
double of pits. The width of every pit is kept constant $W=10^{-5} B$ while the initial depth of the pits $D$ and the distance between two pits $L$ can be varied.

The body is considered to be in plane strain, and finite strain formulation of the kinematics is used [20]. If a material point with coordinates $\left(X_{1}, X_{2}\right)$ in the body undeformed configuration obtains, under the deformation, a position with coordinates $\left(x_{1}, x_{2}\right)$, the deformation gradient is a tensor with components

$$
F_{i j}=\frac{\partial x_{i}}{\partial X_{j}}, \quad i, j=1,2 \text {. }
$$

The Jacobian of the deformation gradient is given by $J=\operatorname{det}\left[F_{i j}\right]$. The constitutive relationship for the body material is of neo-Hookean type. The components of the Cauchy stress tensor at a body interior point are defined as

$$
\sigma_{i j}=\frac{\mu}{J}\left(F_{i j} F_{j i}-\delta_{i j}\right)+\frac{\lambda}{J}(\ln J) \delta_{i j}, \quad i, j=1,2,
$$

where $\mu$ and $\lambda$ are the small strain Lame coefficients of the material, and $\delta_{i j}$ are the components of the Dirac delta function. The body is in equilibrium with the externally applied loads if

$$
\frac{\partial \sigma_{i j}}{\partial x_{i}}=0, \quad i, j=1,2 \text {. }
$$

The components of the displacement and the traction vectors are denoted by $U_{1}$ and $U_{2}$, and $T_{1}$ and $T_{2}$, respectively. The boundary conditions over one load cycle, $0<t<T$, are given by

$$
\begin{aligned}
& T_{1}=T_{2}=0, \quad \text { at } X_{1}=0 \text { and } X_{1}=B \text { and }\left|X_{2}\right|<B, \\
& \mid \begin{array}{l}
T_{1}=0 \\
U_{2}=-\varepsilon_{f} B\left[H(t)-H\left(t-T_{0}\right)\right]
\end{array} \quad \text { at } 0 \leq X_{1} \leq B \text { and } X_{2}=-B, \\
& \mid \begin{array}{l}
T_{1}=0 \\
U_{2}=\varepsilon_{f} B\left[H(t)-H\left(t-T_{0}\right)\right]
\end{array} \quad \text { at } 0 \leq X_{1} \leq B \text { and } X_{2}=B .
\end{aligned}
$$

The magnitude of the applied displacements during the cycle peak, $\varepsilon_{f} B$, ensures a constant strain field in a rectangular body without surface flaws, which is exactly on the threshold to break the protective film of the flat surface. Any small deviation from a flat surface, however, will create sufficient strain concentration to break the film and start the process of strain-driven corrosion. The introduced one ore two surface pits are also assumed traction free, as well as the subsequently evolving surface.

\section{Numerical solution}

A finite element program for finite strain continuum analysis [21] is used to solve the boundary value problem involving eqs.(5)-(8) defined over one cycle. The evolving parts of the surface are described using cubic B-splines [22], which are reshaped after each cycle by displacing the surface nodes according to eq. (3). In order to capture and describe with an acceptable accuracy the new shape, a surface re-meshing algorithm is implemented. It uses ideas from active contours [23] to dense the nodes in the regions of large stretch and curvature. Re-meshing process for the bulk of the body is also an inevitable part of the simulation, since the analysis should follow the evolution of a pit and possible subsequent crack growth at considerable distances. Mesh generation is 
performed by an inbuilt Delauney-type triangulation procedure [24]. Constant strain triangular finite elements are used in the analysis. The unstructured meshes obtained in the generation have a valuable feature. They introduce certain non-homogeneity in the material. This would give a small, but sufficient priority to one of several pits in their competition to attract more of the mechanical energy introduced into the system.

The simulation procedure for one load cycle follows the steps:

- Apply the load and obtaining the mechanical equilibrium of the body;

- Calculate the amount of film damage, $\varepsilon_{s}$, and the surface advancement, $d_{s}$, at each node along the contact surface;

- Update the unloaded configuration by surface reshaping and re-meshing; and

- Re-mesh the updated configuration of the body and accepting it as a reference configuration for the next time interval.

A thorough discussion of the numerical procedure may be found in [13].

\section{Results}

\subsection{Single pit}

Calculations for a single pit on the contact surface have been made for a series of depth to width aspect ratios, ranging from 0.01 to 1.0 . Figure 3(a) shows the penetration advance at the cavity apex (growth rate per cycle, $d / d_{0}$ ); and Fig. 3(b) the cavity extension $(a / W)$ for the aspect ratio $D / W=0.1$. The plots involve the number of simulation cycles $N$. The shapes of the curves in these figures are typical for all initial coefficients of roughness. Moreover, the limiting growth rate, $d_{l i m}$, observed in Fig. 3(a) has been found to have the same value for all configurations. An important characteristic of all curves is the inflection point, denoted by $\mathrm{C}_{1}$ where the maximum acceleration is registered. Point $\mathrm{C}_{2}$ in Fig. 3(a) denotes the offset of steady-state growth, $N_{\text {lim }}$, after which the crack maintains a self-similar profile. The cavity extension at point $\mathrm{C}_{2}$ is denoted by $a_{\text {lim }}$. The region of the body close to the surface pit is shown in Figs. 4(a) and $4(\mathrm{~b})$ at the moments of time corresponding to the points $\mathrm{C}_{1}$ ) and $\mathrm{C}_{2}$, respectively.

Introduce the term incubation time, as the number of numerical steps spent up to the inflection point and denote it by $N_{i n c}$. The corresponding cavity extension at the same position is denoted by $a_{i n c}$. Figure 5 (a)shows the incubation time of the cavity and Fig. 5 (b) the extension at incubation as functions of the initial pit aspect ratio, $D / W$. Fig. 5(a) is plotted against the negative decimal logarithm of the aspect ratio and reveals a linear relationship between $N_{i n c}$ and $\log (D / W)$. Similarly, linear relationship is observed in Fig. 5(b) between the cavity extension at incubation $a_{\text {inc }}$ and the initial pit aspect ratio. The time to the offset of steady-state growth $N_{l i m}$ depends also linearly on the logarithm of the initial pit aspect ratio and is not shown here. An interesting observation is that the normalised length of all cracks at the offset of steady-state $a_{\text {lim }} /$ $W$ is approximately equal to two.

\subsection{Double pits}

Calculations for double pits have been performed for five initial distances between the pits with $L / W=2,3,4,5,10$. In all cases the initial aspect ratio of one of the pits is kept constant $D_{0} / W=0.1$. The depth of the other pit has been varied from $D=D_{0}$ to $D$ 
$=L / 2$. In case of initially different pits, a natural priority is given to the deeper one to grow faster, leading eventually to arrest of the shallower. If the pits are initially identical the mesh irregularity is expected to introduce priority to one of the pits over the other. The case $L / W=4$ is chosen for illustration. Figure 6(a) shows the evolution of the growth rates and Fig. 6(b) the crack extensions for two initially identical pits, i.e. $D=D_{0}=0.1 \mathrm{~W}$. In both plots the solid lines represent the eventually growing crack, while the dashed lines represent the crack that has been arrested. Additionally, the dotted line in Fig. 6(b) represents the normalised crack extension for a single pit with initial aspect ratio 0.1. Figures 7 offer the same information as in Figures 6, but for $D$ / $W=0.5$. Two characteristic points might be identified on these curves. The first one, denoted by $\mathrm{C}_{3}$ is the point where the behaviour of the arrested crack starts deviating from the behaviour of a single crack with the same initial pit aspect ratio. This point might be named a detection point, since at that moment the influence between the two cracks becomes significant. The second characteristic point, denoted by $\mathrm{C}_{4}$, is the moment of arrest of one of the cracks. Figure 8(a) shows the point $\mathrm{C}_{3}$ (a) and Fig. 8(b) the point $\mathrm{C}_{4}$ (b) for $\mathrm{D} / \mathrm{W}=0.5$. The length of the arrested crack is denoted by $a_{a r r}$. Figure 9 shows the lengths of the arrested cracks as functions of the ratio between the two initial pit depths for all considered initial distances between the two pits. Points on the curves, corresponding to $10 D_{0}<D<L / 2$ for $L>2 W$ are left outside the plot. If the normalised lengths of the arrested cracks are plotted versus $D / L$, the five curves collapse approximately onto a single curve.

\section{Discussion}

The experiments performed for a single surface pit configuration aimed at obtaining a general picture of the cavity evolution under the introduced strain-driven corrosion model. The typical behaviour of the growth rate, as illustrated in Figure 3 (a), shows three distinct stages of growth. In the first stage strain and penetration localization occurs, leading to growth acceleration. This stage finishes at the point of maximum acceleration, where the cavity assumes a profile with the smallest radius of curvature during the entire evolution, see Figure 4(a). Therefore we call the first stage crack incubation. The second stage is characterised by a growth with decreasing acceleration and a blunting profile at the cavity apex. Finally a steady-state growth is reached, see Figure 4(b), at a growth rate, which is found to be independent of the initial pit geometry. The value of the steady-state growth rate, which is read in Figures 3(a), 6(a) and 7(a), together with equation (1) demonstrates that the self-similar profile maintained at the third stage is characterised by a constant strain at the apex approximately ten times larger than the remotely applied strain. An interesting observation is that both times to incubation and times to steady-state are proportional to the logarithm of the initial pit depths, where the first relationship is shown in Figure 5(a). The incubation lengths depend linearly on the initial pit depths, see Figure 5(b), while the crack lengths at the offset of a steady-state are found independent of the pit depths. Thus, the approximately self-similar profile is reached at a length of about two times the initial pit width and is characterised by a strain concentration factor of ten. The proportionality constants depicted in Fig. 5(a) and 5(b) should be taken with caution, since they probably depend on the initial pit profile, not only the ratio between the depth and the width. The initial profiles used in this study were always described by cubic polynomials and a change to second-degree curves would possibly reflect the values 
given in these figures. The linear relationships, however, could be used as benchmarks in performing laboratory experiments and if supported by them only two different pit depths would be enough to be tested in the future.

The configurations with two pits were investigated to obtain a notion of cavities competition, as well as to study the influence of the distance between the pits and the difference in their initial depths. Four stages of growth rate evolution could be distinguished from Figures 6(a) and 7(a). In the beginning each cavity develops independently showing the behaviour of a single pit evolution. This first stage finishes at the moment, where the two cavities start interacting. They are point $\mathrm{C}_{3}$ depicted in Figures 6 and 7. The configuration at the detection point is shown in Figure 8(a) for a selected parameters setting. In the second stage the two cavities change their growth path by repulsion from each other and the growth rates of both of them slow down compared to the rate of a single pit with the same initial depth. This observation shows that having competing pits, two or any number, is preferable than having a single pit. If the depths of the two pits are initially equal or nearly equal, the second stage is characterised by a common plateau in the growth rate, see Figure 6(a). For large differences in initial depths a plateau is not observed, but the second stage finishes at a point of maximum growth rate, see Figure 7(a). The appearance of a plateau was not observed to depend on the distance between the pits, but only on the ratio between their initial depths. The level at which the plateau occurs, however, depends on the distance between the pits. For closely situated pits, $L=2 W$, with short times to detection, the plateau value is nearly equal to $d_{\lim } / \sqrt{ } 2$. This result is expected since the two cavities should share the supplied strain energy approximately equally. With increase of the distance between the initial pits, the plateau value increases and for distant enough pits it reaches the steady-state growth rate. This situation was observed for $L=10 \mathrm{~W}$ and is due to the fact that the point of detection has not been reached before steady-states for both cavities has been established. The third stage of the evolution is characterised by decreasing growth rate of one of the cavities and finishes at its arrest (point $\mathrm{C}_{4}$ depicted in Figures 6 and 7). Figure 8(b) presents the region of interest at the moment of arrest. While the growth rate of the eventually arrested cavity decreases, the growth rate of the eventually growing one increases and reaches the steady-state value, $d_{l i m}$, approximately at the time of arrest. This situation is again changed for large distance between the pits, $L=10 \mathrm{~W}$, where the first stage of evolution actually finishes after certain distance of steady-state growth. At the point of detection, both cavities assume decreasing growth rates during the second stage. The third stage starts when the growth rate of one of the cavities increases again to the steady-state value, while the other one continues growing with decreasing rate to final arrest.

The result, presented in Figure 9, shows the relative lengths of the arrested cracks as a function of the ratio between the initial pits depths. An interesting outcome of the simulations is the fact that for small differences between the initial pits, the relative lengths of the arrested cracks turned out to be almost independent of the distance between the pits. For equal or nearly equal initial pit depths, the arrested crack lengths are found to be about 2.5 times the distance. For large ratios between the initial pit depths the influence of the distance becomes pronounced with larger lengths at arrest for more distant pits. The curves, given in Figure 9, could be used for obtaining the distribution of arrested cracks on a surface. For example, if a growing crack is observed, then it could be estimated that the longest possible arrested crack at distance $L$ has a length of about $2.5 \mathrm{~L}$. All the cracks between them must be arrested earlier and have 
shorter lengths. A possible way of simulating surface cracks distribution is through statistical simulation. It has to be performed by varying both the distance and the pit depths ratios if large ratios have to be considered. As an example, a normal distribution of the ratio, $D / D_{0}$, is depicted by stars above the horizontal axis in Figure 9 . The corresponding lengths of the arrested cracks, for the case $L=4 W$, are given with crosses to the right of the vertical axes in the figure. Extending this approach by varying the distance would create a statistical picture of the arrested crack lengths on a surface.

7. Conclusions

Finally, two essential limitations of the model will be mentioned. The first concerns with electrochemical effects. Equation (2) is approximately valid for moderately deep cavities, where no localization of the environmental composition has occurred and the chemistry could be assumed the same as in the bulk solution. When the cavity or crack becomes deeper, the transport processes in the solution, like diffusion and electromigration of various species, must be considered when accounting for the local environment close to the crack tip. This issue present no serious difficulties and might be incorporated in a future extension of the model. However, a large part of the cavity growth followed in this work may be accepted as not effected by this limitation.

The second limitation to the model is posed by the assumption of material's homogeneity. In case of intergranular stress-corrosion crack growth the distance between the grain boundaries, or inter-phase thickness, would set a restriction to the blunting of the tip which is observed after the incubation period in the present study. Therefore, the self-similar shape of the tip at steady-state could not be maintained indefinitely. If a crack, at some stage of growth, has a tip opening smaller than the interphase thickness, then when the blunting tip hits the grain boundaries an acceleration of the growth would probably occur. It is also possible that a new steady-state condition of growth might not be reached again. If, from another side, the inter-phase thickness is small enough compared to the initial pit size, then the evolution during the incubation will be modified. This could also lead to an accelerating crack growth with no steadystate ever assumed.

\section{Acknowledgements}

The financial support for this work, coming from the Swedish Centre for Nuclear Technology (SKC), is highly appreciated.

\section{References}

[1] V. V. Bolotin, Mechanics of Fatigue (CRC Press, Boca Raton, Florida, 1998).

[2] J. D. Atkinson, J. Yu and Z.-Y. Chen, An analysis of the effects of sulphur content and potential on corrosion fatigue crack growth in reactor pressure vessel steels, Corros. Sci. 38 (5), 755-765 (1996).

[3] J. Ruiz and M. Elices, The role of environmental exposure in the fatigue behaviour of an aluminium alloy, Corros. Sci. 39 (12), 2117-2141 (1997).

[4] W. Y. Maeng, Y. H. Kang, T. W. Nam, S. Ohashi and T. Ishihara, Synergistic interaction of fatigue and stress corrosion crack growth behaviour in Alloy 600 in high temperature and high pressure water, J. Nucl. Mater. 275, 194-200 (1999). 
[5] M. E. Hoffman and P. C. Hoffman, Corrosion and fatigue research - structural issues and relevance to naval aviation, Int. J. Fatigue 23, S1-S10 (2001).

[6] D. V. Ramsamooj and T. A. Shugar, Modeling of corrosion fatigue in metals in an aggressive environment, Int. J. Fatigue 23, S301-S309 (2001).

[7] J. Congleton, E. A. Charles and G. Sui, Review on effect of cyclic loading on environmental assisted cracking of alloy 600 in typical nuclear coolant waters, Corros. Sci. 43, 2265-2279 (2001).

[8] R. N. Parkins, Current understanding of stress-corrosion cracking, JOM-J. Min. Met. Mat. S. December, 12-19 (1992).

[9] A. Turnbull, Modelling of environment assisted cracking, Corros. Sci. 34 (6), 921-960 (1993).

[10] F. P. Ford and P. L. Andresen, Stress corrosion cracking of low alloy steels in $288^{\circ} \mathrm{C}$ water, Corrosion '89, NACE, Houston, Paper No. 498, 1-19 (1989).

[11] F. P. Ford, Quantitative prediction of environmentally assisted cracking, Corrosion 52 (5), 375-395 (1996).

[12] G. R. Engelhardt, D. D. Macdonald and M. Urquidi-Macdonald, Development of fast algorithms for estimating stress corrosion crack growth rate, Corros. Sci. 41, $2267-$ 2302 (1999).

[13] A. P. Jivkov and P. Ståhle, Strain-driven corrosion crack growth: A pilot study of intergranular stress corrosion cracking, Eng. Fract. Mech. 69 (18), 2095-2111 (2002).

[14] G. S. Chen, K.-C. Wan, M. Gao, R. P. Wei and T. H. Flournoy, Transition from pitting to fatigue crack growth - modelling of corrosion fatigue crack nucleation in a 2024-T3 aluminium alloy, Mat. Sci. Eng. A219, 126-132 (1996).

[15] S. I. Rokhlin, J. Y. Kim, H. Nagy and B. Zoofan, Effect of pitting corrosion on fatigue crack initiation and fatigue life, Eng. Fract. Mech. 62, 425-444 (1999).

[16] P. S. Pao, S. J. Gill and C. R. Feng, On fatigue crack initiation from corrosion pits in 7075-T7351 aluminium alloy, Scripta mater. 43, 391-396 (2000).

[17] G. Murtaza and R. Akid, Empirical corrosion fatigue life prediction models of a high strength steel, Eng. Fract. Mech. 67, 461-474 (2000).

[18] K. K. Sankaran, R. Perez and K. V. Jata, Effects of pitting corrosion on the fatigue behaviour of aluminium alloy 7075-T6: modelling and experimental studies, Mat. Sci. Eng. A297, 223-229 (2001).

[19] G. Engelhardt, M. Urquidi-Macdonald and D. D. Macdonald, A simplified method for estimating corrosion cavity growth rates, Corros. Sci. 39 (3), 419-441 (1997).

[20] J. Bonet and R. D. Wood, Nonlinear continuum mechanics for finite element analysis (Cambridge University Press, Cambridge, 1997).

[21] A. P. Jivkov, MATERIA - a finite strain based finite element program (Development Report, Solid Mechanics, Malmö University, 2000).

[22] G. D. Knott, Interpolating cubic splines (Progress in computer science and applied logic, Birkhauser, Boston, 1999).

[23] J. A. Sethian, Level Set Methods and Fast Marching Methods: Evolving Interfaces in Computational Geometry, Fluid Mechanics, Computer Vision, and Materials Science (Cambridge Monographs on Applied and Computational Mathematics, Cambridge University Press, 1999).

[24] J. R. Shewchuk, Delaunay refinement algorithms for triangular mesh generation, Comput. Geom. - Theory Appl. 22, 21-74 (2002). 


\section{Figure captions:}

Figure 1. Schematic of the passive film behaviour.

Figure 2. Schematic of single pit geometry (a) and couple of pits geometry (b).

Figure 3. Normalised crack growth rate (a) and crack extension (b) for $D / W=0.1$.

Figure 4. Near pit region at maximum acceleration (a) and at constant growth rate (b) for $D / W=0.1$.

Figure 5. Incubation time (a) and incubation length (b) versus initial pit aspect ratio.

Figure 6. Normalised growth rates (a) and crack extensions (b) for $D=D_{0}$ and $L=4 W$.

Figure 7. Normalised growth rates (a) and crack extensions (b) for $D=5 D_{0}$ and $L=4 \mathrm{~W}$.

Figure 8. Near pits region at detection (a) and at arrest (b) for $D=5 D_{0}$ and $L=4 W$.

Figure 9. Relative arrest lengths versus pit depths ratio for $L / W=2,3,4,5,10$. 


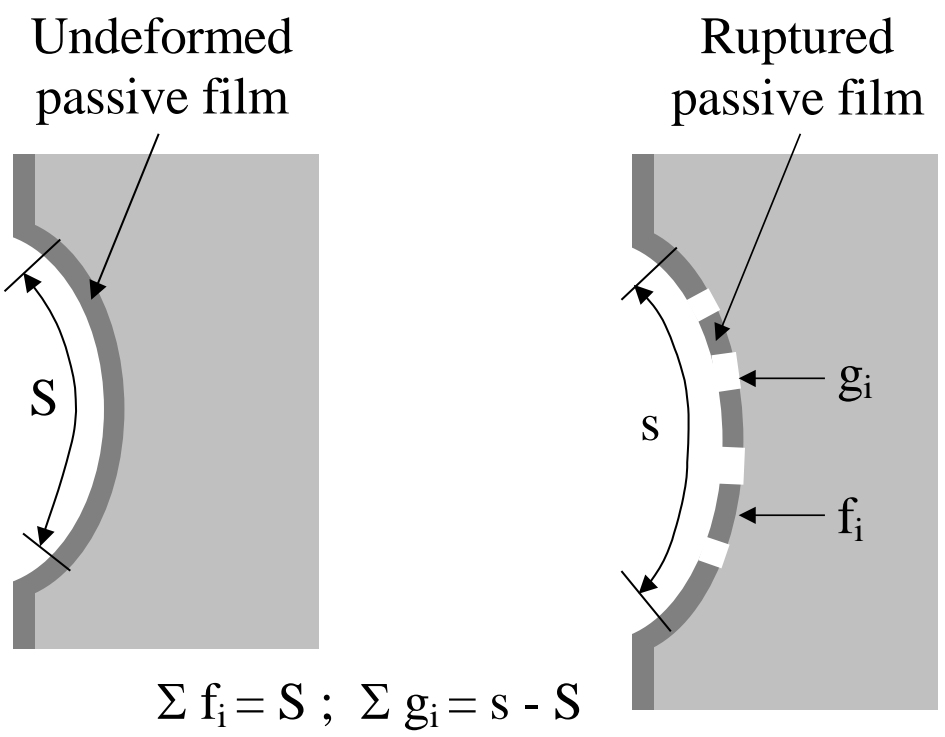

Figure 1. 

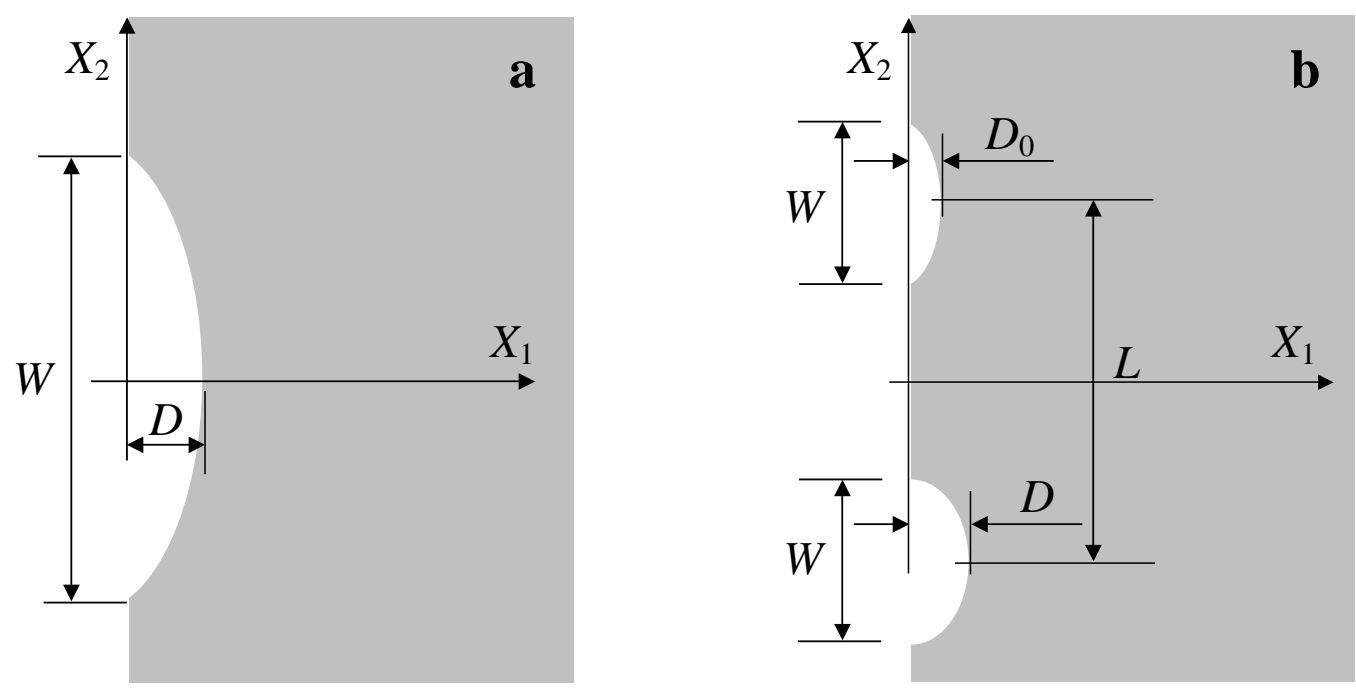

Figure 2. 

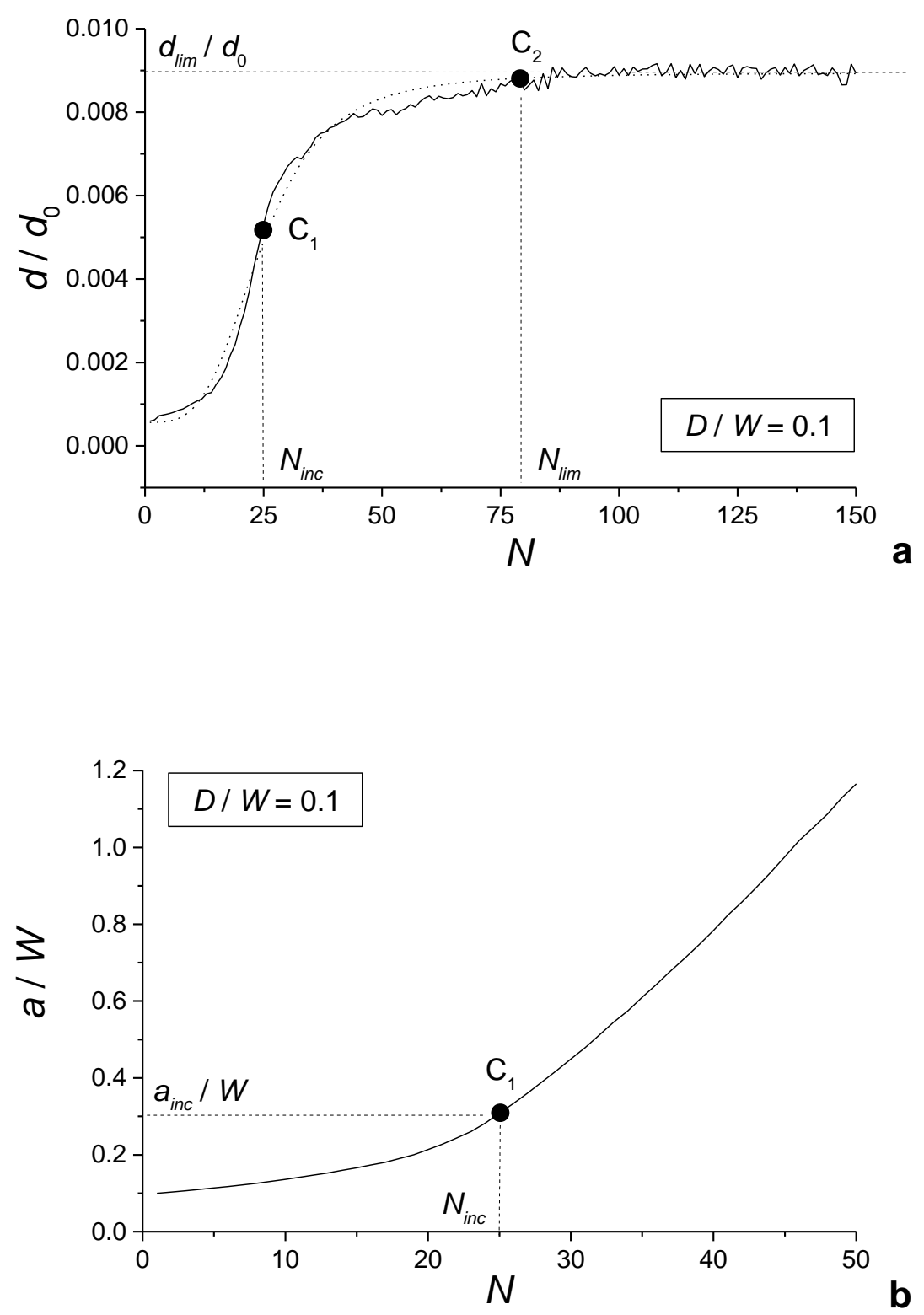

Figure 3. 

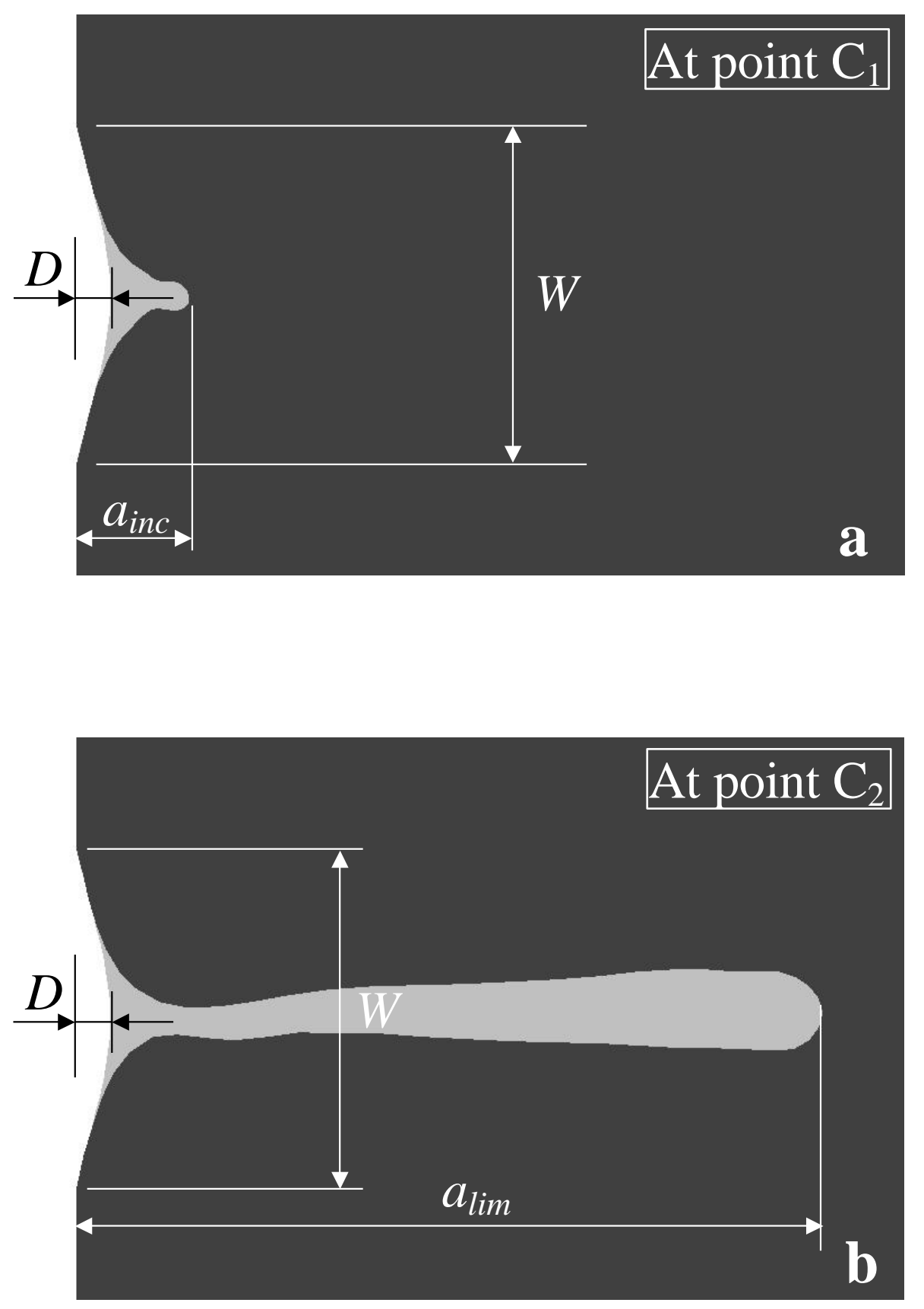

Figure 4. 

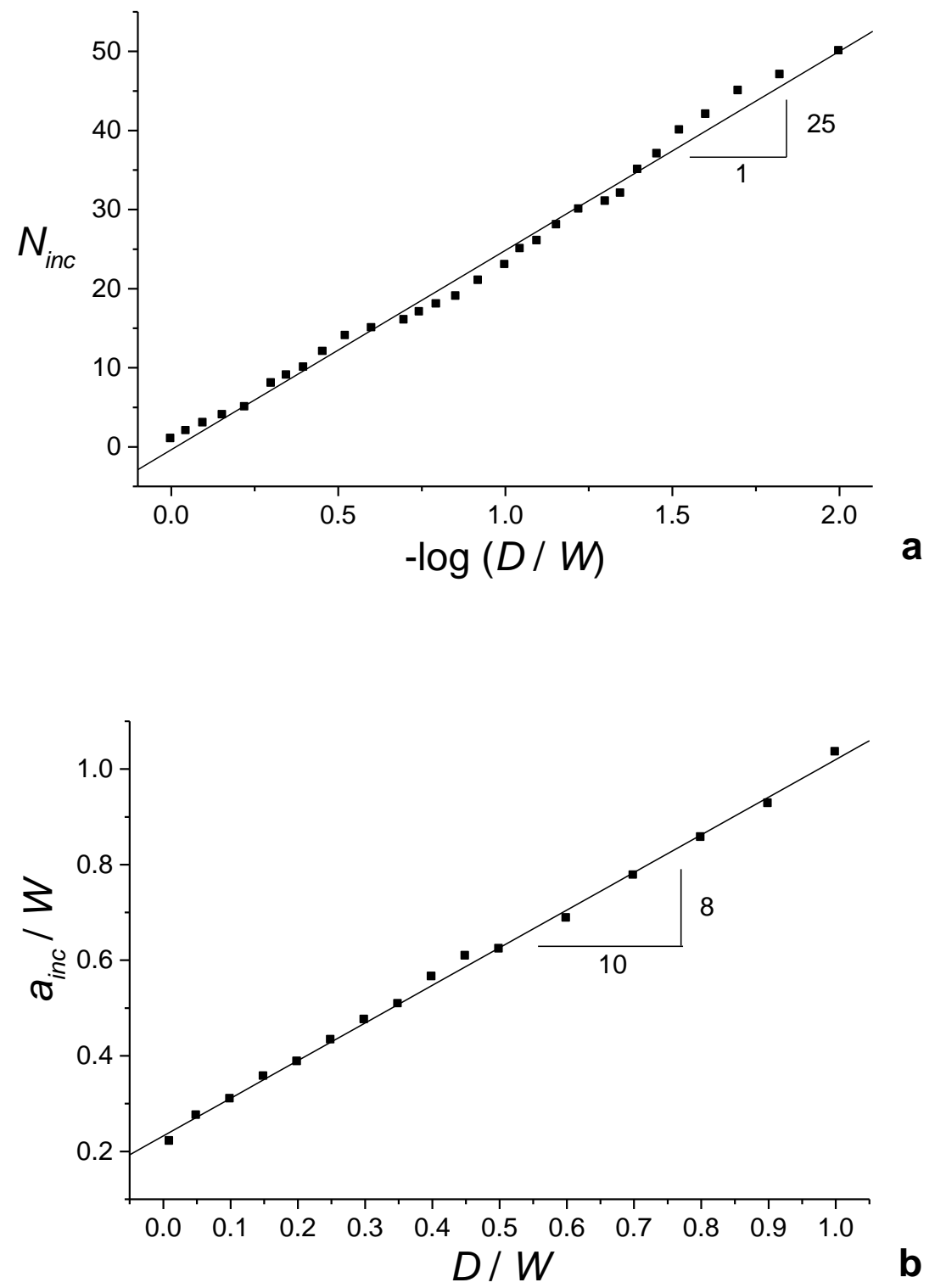

Figure 5. 

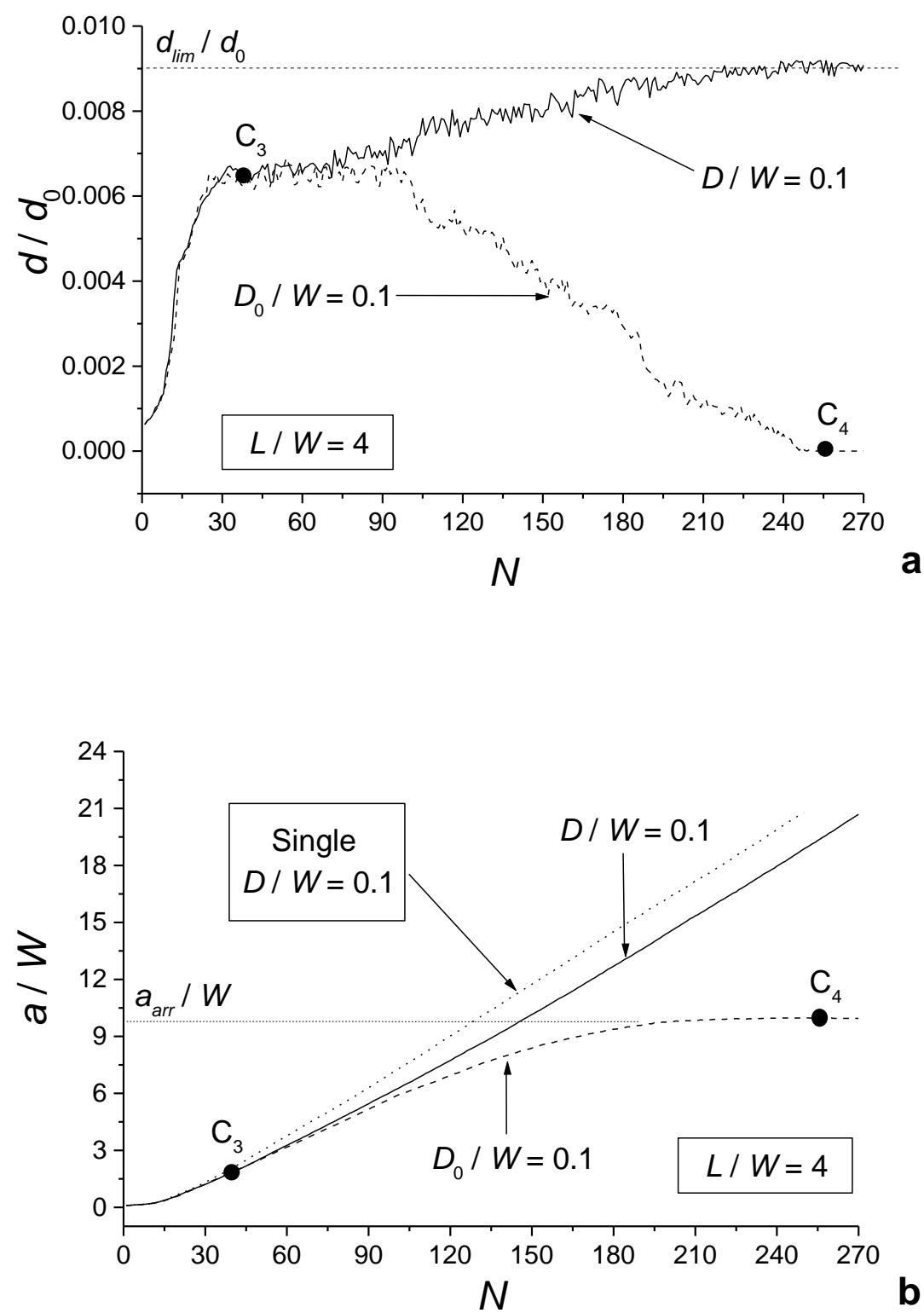

Figure 6. 

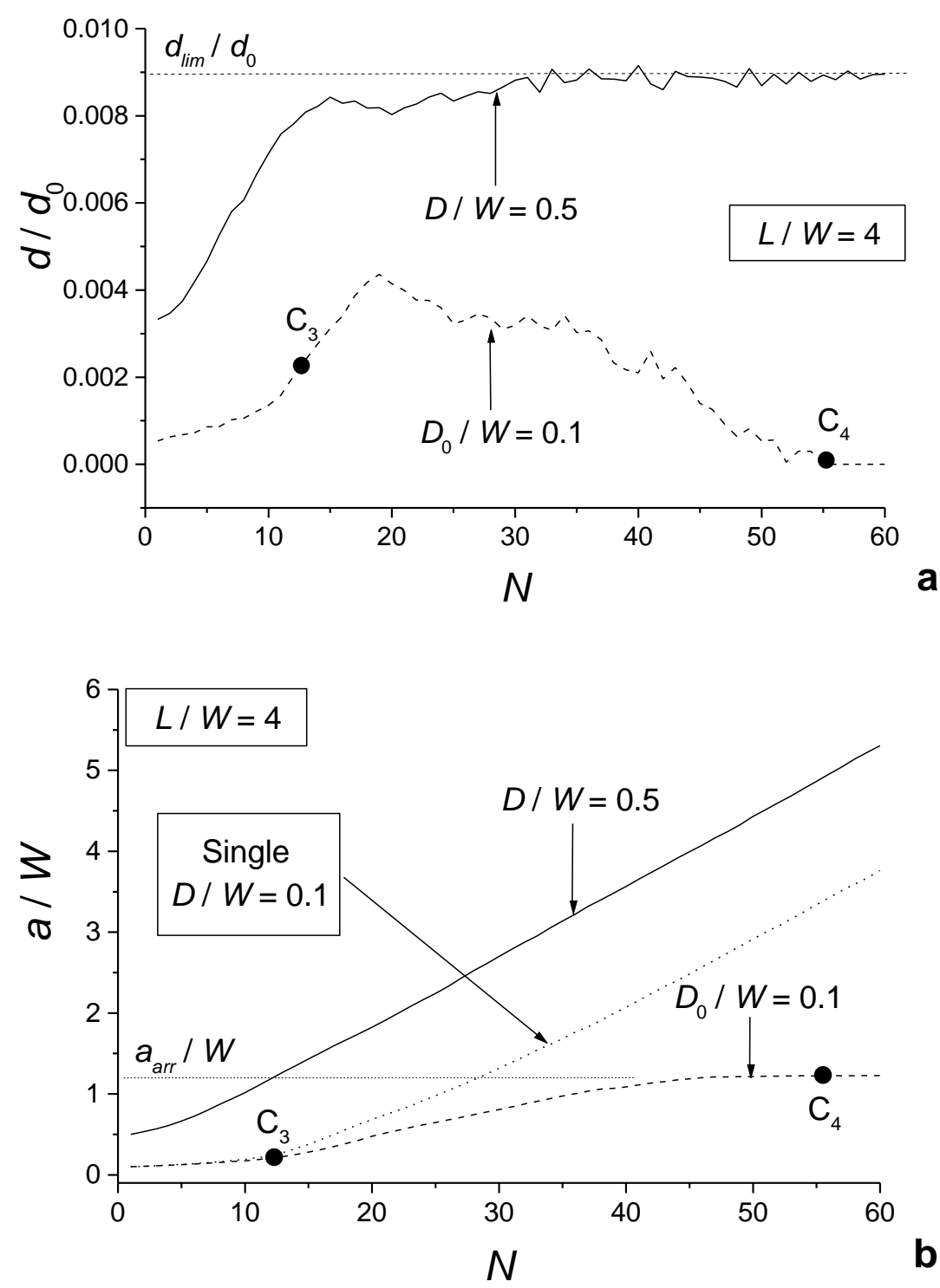

Figure 7. 


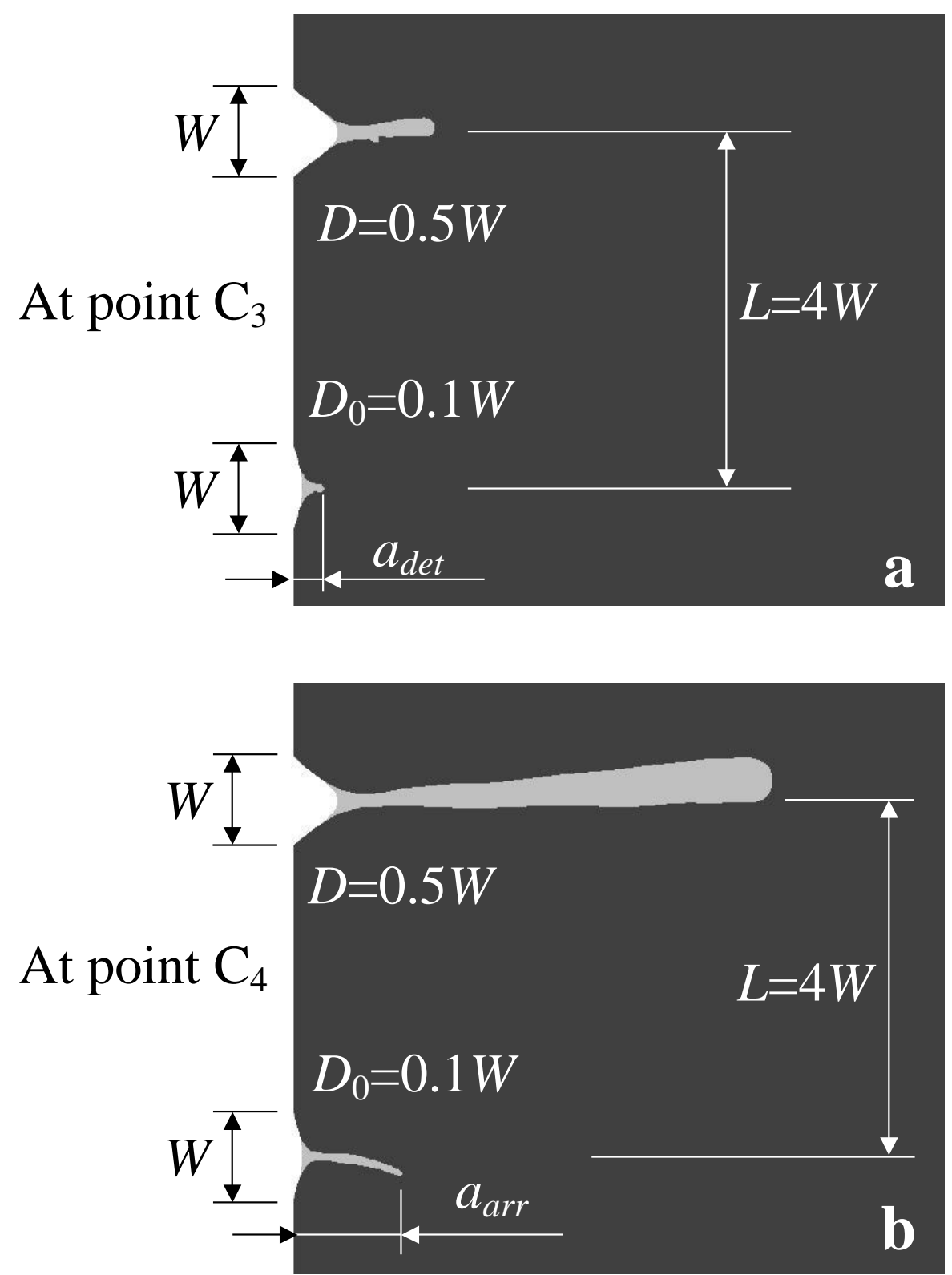

Figure 8. 


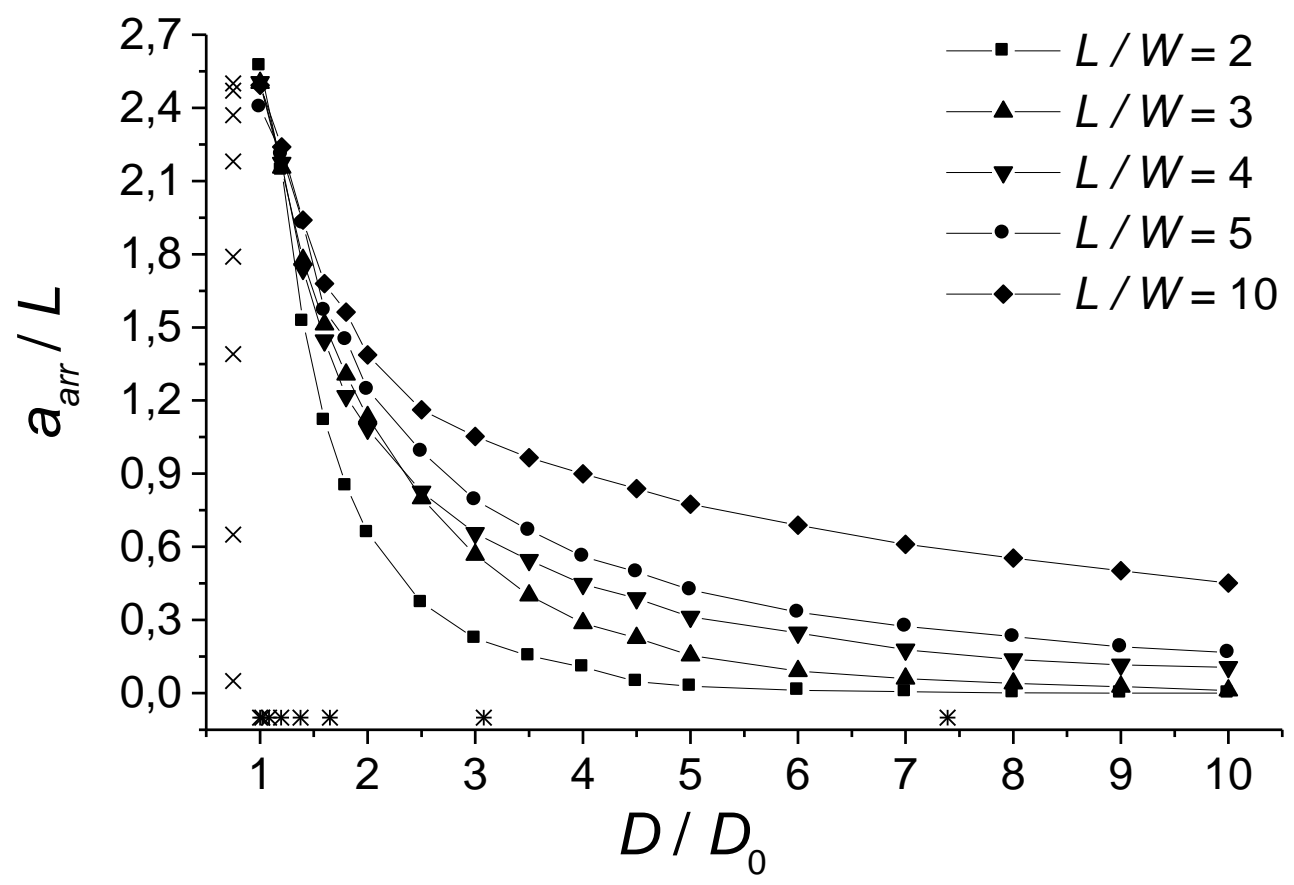

Figure 9. 